\title{
Chip-scale Classical and Quantum Nonlinear Photonic Mixers (Project Report 1201308-Y1)
}

\section{SHAYAN MOOKHERJEA}

Department of Electrical and Computer Engineering, University of California, San Diego, MC 0407 La Jolla CA 92093-0407 USA

Email:smookher@ucsd.edu

\section{Introduction and Goals}

The goal of this NSF-funded project [1201308 - Year 1] is to develop an integrated photonics device technology for compact and efficient nonlinear classical and quantum photonic mixers. The principal focus of research during the first year (2012-13) was on designing devices using a foundry-fabrication silicon photonics platform, which is rapidly growing in importance both in university research and in industry.

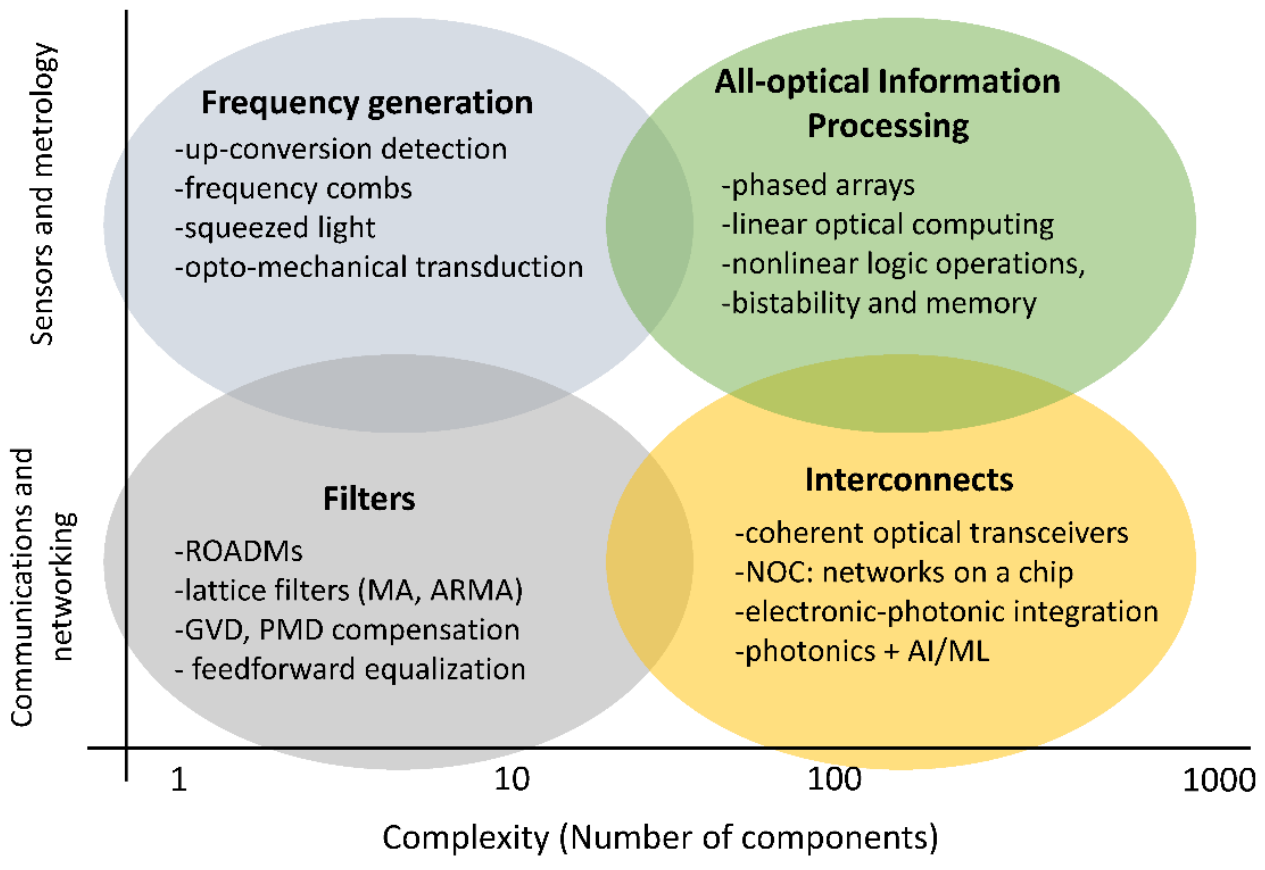

Figure 1 Progress in applications of integrated photonics (communications, networking, sensors and metrology) driven by advances in scalability and complexity of photonic circuits.

A silicon photonics platform consisting of single-mode optical waveguides, microrings and coupled-resonator waveguides was used to study topics in nonlinear and quantum photonics (see Section 2). These topics gained in practical importance with the emergence of silicon photonics as a planar lightwave circuit (PLC) technology for integrated communications devices, and the growing need for wavelength-agile communications device that use the optical spectrum efficiently. 
There are significant opportunities and challenges in using silicon photonics for nonlinear optics at telecommunications wavelengths. Optical nonlinearity can affect several components that are relevant to this project, such as racetrack resonators [1]. There are observable nonlinear optical effects in single-mode silicon waveguides at a few tens of milliwatts of optical power, because of the materials properties of silicon, and the high degree of confinement of the optical mode with a small mode cross-sectional area. In resonators, nonlinear effects are easily observed because of the resonant enhancement of the optical intensity. In the devices that were studied in this project, the most dominant nonlinear impairments were from free-carrier absorption (FCA) losses due to two-photon absorption (TPA) generated carriers (electrons and holes) [2]. These losses were partially mitigated by reducing the free-carrier lifetime through a reverse-biased $\mathrm{p}-\mathrm{i}-\mathrm{n}$ diode fabricated across the waveguide crosssection [3]. Such "active" waveguides were also used as the building blocks for microring resonators and coupled-resonator optical waveguides (CROWs) [4], which may be useful for filters [5], tunable dispersion devices and lasers [6], [7].

Another set of challenges encountered by designers is related to the sensitivity of resonators in high-index contrast materials to fabrication disorder, which can affect the waveguiding properties of CROWs [8]. A number of ideas were studied and evaluated for feasibility including precision post-fabrication trimming [9], [10]. An accurate design of the waveguide-resonator coupling is important for CROWs and often requires detailed simulations, since there can be significant deviations from conventional coupled-mode theory in the highindex-contrast regime of silicon photonics [11]. In preparatory work to this project, silicon microring CROWs consisting of up to 235 coupled resonators were fabricated and measured [12].

\section{Activities}

We designed devices for fabrication in a multi-project wafer (MPW) run hosted by IME in Singapore. This was a custom run in which we worked directly with foundry staff to identify and customize suitable processing steps. The special steps were mainly related to roughness-reduction processing, since sidewall roughness increases optical propagation loss. Loss reduction is critically important in nonlinear optical devices, since it affects each of the interacting waves in an exponential manner. For example, $1 \mathrm{~dB}$ additional propagation loss at the pump and signal waves reduces the idler power generated by four-wave mixing by $3 \mathrm{~dB}$, and the idler itself experiences greater propagation loss once generated. 
In order to design microring CROWs to a target free-spectral range (FSR), the dispersion of the structure must be carefully simulated. The dispersion of directional couplers was studied and experimentally verified [13]. An analytical expression was derived for the coupling dispersion to help guide the design of subsequent devices.

To help in measurements, a method of quantitative infrared imaging of the device-under-test which we had developed in an earlier project [14] was extended to achieve higher dynamic range [15]. Multiple images were acquired at different exposure settings and stitched together using software (this is known as "bracketing" in photography) to achieve a resulting image that has more dynamic range (bits) than a single raw image.

As one of the outcomes of this research project, we demonstrated continuouswave $(\mathrm{CW})$ four-wave mixing in conventional waveguides with carrier sweepout (see Figure 2) and in microring resonators [16], [17]. CW wavelength conversion with an unmodulated pump is desirable for optical communications because FWM is format-independent, i.e., the data modulation on the input signal is translated to the newly-generated idler wavelength. As shown in Fig. 2 , a high level of conversion was achieved by using carrier sweepout, with slightly less than $10 \%$ conversion efficiency, over a fairly wide bandwidth (20 $\mathrm{nm})$ at moderate input pump power $(60 \mathrm{~mW})$ compared to previous results. Even higher conversion efficiency may be achieved by improving the pump coupling efficiency to support higher pump powers. Some waveguides were damaged during testing, because of the dissipation of significant amounts of pump power near the input facet. The design of efficient waveguide couplers for high-power coupling to silicon waveguides from optical fiber remains an open challenge.

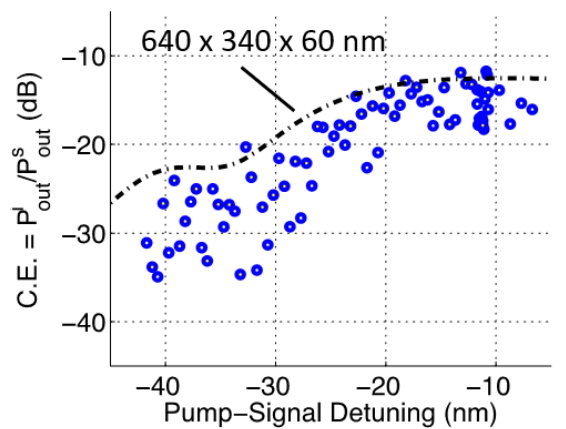

Max Conv. Efficiency: $-12 \mathrm{~dB}$ upto $20 \mathrm{~nm}$ detuning Pump power: $60 \mathrm{~mW}$

Figure 2 Results on Four-Wave Mixing in silicon waveguides
We demonstrated wavelength conversion using nonlinear optics in a coupledresonator optical waveguide (CROW). A CROW provides resonant enhancement of the conversion efficiency [18], [19], but also offers a relatively wide passband (up to several nanometers), unlike a single resonator. This can be very helpful in practical use, since a narrow-band resonator can drift easily due to environmental conditions or changes in the optical power. An analytical treatment of pulse propagation has been developed for such structures, which clearly shows the role played by slow light [20], [21] and 
such models were used for preliminary estimates of the device performance for designs prepared in this project. One of the long-standing concerns of the CROW device configuration is the relatively high dispersion of the structure [22], which can impact nonlinear conversion efficiency and other parameters of interest [23]-[26].
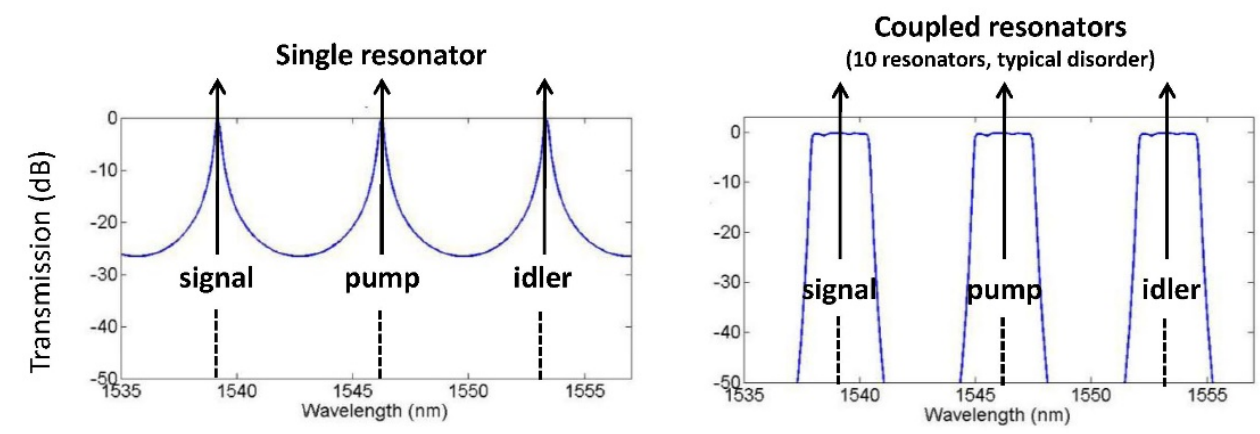

Figure 3 Calculated transmission spectrum of a single resonator (left panel) and coupled
resonator waveguide (right panel) as intended for use in four-wave mixing. The flat passbands
of the latter are useful to retain the advantages of resonant enhancement of the nonlinearity even
when there are minor variations in the operating conditions which cause fluctuations in the
precise resonant wavelength.

\section{Progress and Impact}

Nonlinear wavelength conversion is based on an ultrafast modification of the refractive index. This method overcomes some limitations of other tuning techniques in silicon photonics. For example, thermo-optic tuning is slow [27], and can lead to significant crosstalk among devices unless they are widely separated. On the other hand, for wavelength conversion over relatively short frequency ranges (less than $100 \mathrm{GHz}$ ), an all-electro-optic approach may be simpler and more convenient. High-bandwidth integrated electro-optic modulators fabricated using thin-film ferroelectric materials bonded to silicon wafers were demonstrated in subsequent research [28].

Disorder is a topic of significant concern in CROWs, since it can lead to a variety of complex phenomena such as localization of light. However, one potential advantage of structures designed in a semiconductor such as silicon is that localization can be controlled electronically, which is not possible in dielectric insulator materials [29]. This may lead to new insights into how to harness disorder which cannot be eliminated.

Impact on Human Resources: Two graduate students and one undergraduate student were supported by NSF funds and worked on device design, fabrication and testing. We gratefully acknowledge NIST (Gaithersburg, MD) in collaborating with us in performing some measurements under and NSFsupported supplement. 


\section{Subsequent Extensions}

This project demonstrated four-wave mixing in silicon waveguides, microrings, and CROW structures. Wavelength conversion in optical networks needs additional components for practical use. Additional work has to be done to design wavelength selective combiners / splitters that can multiplex and demultiplex pump wavelengths from signal and idler wavelengths [30]. The insertion loss of coupling light from fibers to waveguides also has to be improved. Methods of dealing with state-of-polarization variations in practical networks have to be developed. These challenges are also manifest in linear optics applications, such as add/drop multiplexing and filtering.

The ability to rapidly tune wavelength conversion can be helpful in realizing agile optical networks. Although the thermo-optic effect is considered to be quite slow (typically, in the milli-second regime), later research showed that it is possible to design silicon photonic devices which can achieve a substantially faster thermo-optic tuning, in the micro-second regime [31]. However, these devices had more optical propagation loss than is desirable for nonlinear optics.

A variant of four-wave mixing in which only the pump is input to the device is known as spontaneous four-wave mixing (SFWM). This can be used to generate correlated photon pairs at the signal and idler wavelengths, in a manner that is similar to spontaneous parametric down-conversion (SPDC). Whereas SPDC is usually performed using nonlinear crystals (or thin-films of nonlinear crystals [32]), SFWM can be performed using devices fabricated entirely using silicon [33]. Silicon waveguides with $\mathrm{p}-\mathrm{i}-\mathrm{n}$ junctions fabricated across them in this project were useful in generating entangled photon pairs through SFWM [34]. Research performed in the first year of this project laid the groundwork for a detailed design and device optimization which eventually led to a significant improvement in the pair-generation properties of silicon microrings [35], [36].

This project addresses several aspects of nonlinear optics which go beyond the traditional assumptions used in integrated optics, such as passive dielectric waveguides, no carrier sweepout and no slow light effects. Physics models for analyzing nonlinear light propagation in the interesting regime that lies beyond these assumptions are under development [37], but device applications are not yet anticipated.

\section{Open-Access Reporting Initiative}

PRAISE: This open-access document is provided in support of our PRAISE

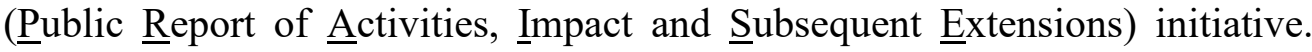


What is it? An open-access document shared with the public which describes the research outcomes of publicly-funded projects. For us, these projects are typically funded by the NSF (National Science Foundation).

\section{References}

[1] S. Mookherjea and M. A. Schneider, "The nonlinear microring add-drop filter," Opt. Express, vol. 16, no. 19, p. 15130, Sep. 2008, doi: 10.1364/OE.16.015130.

[2] K. K. Tsia, S. Fathpour, and B. Jalali, "Energy harvesting in silicon wavelength converters," Opt. Express, OE, vol. 14, no. 25, pp. 1232712333, Dec. 2006, doi: 10.1364/OE.14.012327.

[3] H. Rong et al., "An all-silicon Raman laser," Nature, vol. 433, no. 7023, pp. 292-294, Jan. 2005, doi: 10.1038/nature03273.

[4] F. Morichetti, C. Ferrari, A. Canciamilla, and A. Melloni, "The first decade of coupled resonator optical waveguides: bringing slow light to applications," Laser \& Photon. Rev., vol. 6, no. 1, pp. 74-96, Jan. 2012, doi: 10.1002/lpor.201100018.

[5] S. Mookherjea, "Spectral characteristics of coupled resonators," J. Opt. Soc. Am. B, vol. 23, no. 6, p. 1137, Jun. 2006, doi: 10.1364/JOSAB.23.001137.

[6] S. Mookherjea, "Semiconductor coupled-resonator optical waveguide laser," Appl. Phys. Lett., vol. 84, no. 17, pp. 3265-3267, Apr. 2004, doi: 10.1063/1.1719278.

[7] S. Mookherjea, "Using gain to tune the dispersion relation of coupledresonator optical waveguides," IEEE Photon. Technol. Lett., vol. 18, no. 5, pp. 715-717, Mar. 2006, doi: 10.1109/LPT.2006.871144.

[8] S. Mookherjea and M. A. Schneider, "Avoiding bandwidth collapse in long chains of coupled optical microresonators," Opt. Lett., vol. 36, no. 23, p. 4557, Dec. 2011, doi: 10.1364/OL.36.004557.

[9] Y. Shen, I. B. Divliansky, D. N. Basov, and S. Mookherjea, "Electricfield-driven nano-oxidation trimming of silicon microrings and interferometers," Opt. Lett., vol. 36, no. 14, p. 2668, Jul. 2011, doi: 10.1364/OL.36.002668.

[10] Y. Shen, I. B. Divliansky, D. N. Basov, and S. Mookherjea, "Perfect set-and-forget alignment of silicon photonic resonators and interferometers," in Optical Fiber Communication Conference/National Fiber Optic Engineers Conference 2011, Los Angeles, California, 2011, p. PDPC3. doi: 10.1364/OFC.2011.PDPC3.

[11] M. L. Cooper and S. Mookherjea, "Numerically-assisted coupledmode theory for silicon waveguide couplers and arrayed waveguides," Opt. Express, vol. 17, no. 3, p. 1583, Feb. 2009, doi: 10.1364/OE.17.001583. 
[12] M. L. Cooper et al., "235-ring Coupled-Resonator Optical Waveguides," in Conference on Lasers and Electro-Optics 2010, San Jose, California, 2010, p. CTuHH3. doi: 10.1364/CLEO.2010.CTuHH3.

[13] R. Aguinaldo, Yiran Shen, and S. Mookherjea, "Large Dispersion of Silicon Directional Couplers Obtained via Wideband Microring Parametric Characterization," IEEE Photon. Technol. Lett., vol. 24, no. 14, pp. 1242-1244, Jul. 2012, doi: 10.1109/LPT.2012.2198639.

[14] M. L. Cooper, G. Gupta, J. S. Park, M. A. Schneider, I. B. Divliansky, and S. Mookherjea, "Quantitative infrared imaging of silicon-on-insulator microring resonators," Opt. Lett., vol. 35, no. 5, p. 784, Mar. 2010, doi: 10.1364/OL.35.000784.

[15] S. Mookherjea and H. R. Grant, "High dynamic range microscope infrared imaging of silicon nanophotonic devices," Opt. Lett., vol. 37, no. 22, p. 4705, Nov. 2012, doi: 10.1364/OL.37.004705.

[16] J. R. Ong, R. Kumar, R. Aguinaldo, and S. Mookherjea, "Efficient CW Four-Wave Mixing in Silicon-on-Insulator Micro-Rings With Active Carrier Removal," IEEE Photon. Technol. Lett., vol. 25, no. 17, pp. 1699 1702, Sep. 2013, doi: 10.1109/LPT.2013.2272521.

[17] J. R. Ong et al., "Low-power continuous-wave four-wave mixing in silicon coupled-resonator optical waveguides," Opt. Lett., vol. 36, no. 15, pp. 2964-2966, 2011.

[18] S. Mookherjea and A. Yariv, "Second-harmonic generation with pulses in a coupled-resonator optical waveguide," Phys. Rev. E, vol. 65, no. 2, p. 026607, Jan. 2002, doi: 10.1103/PhysRevE.65.026607.

[19] S. Mookherjea and A. Yariv, "Kerr-stabilized super-resonant modes in coupled-resonator optical waveguides," Phys. Rev. E, vol. 66, no. 4, p. 046610, Oct. 2002, doi: 10.1103/PhysRevE.66.046610.

[20] S. Mookherjea and A. Yariv, "Optical pulse propagation in the tightbinding approximation," Opt. Express, vol. 9, no. 2, p. 91, Jul. 2001, doi: 10.1364/OE.9.000091.

[21] S. Mookherjea and A. Yariv, "Optical pulse propagation and holographic storage in a coupled-resonator optical waveguide," Phys. Rev. $E$, vol. 64, no. 6, p. 066602 , Nov. 2001, doi: 10.1103/PhysRevE.64.066602.

[22] M. L. Cooper and S. Mookherjea, "Modeling of Multiband Transmission in Long Silicon Coupled-Resonator Optical Waveguides," IEEE Photon. Technol. Lett., vol. 23, no. 13, pp. 872-874, Jul. 2011, doi: 10.1109/LPT.2011.2141657.

[23] S. Mookherjea and A. Yariv, "Pulse propagation in a coupled resonator optical waveguide to all orders of dispersion," Phys. Rev. E, vol. 65, no. 5, p. 056601, Apr. 2002, doi: 10.1103/PhysRevE.65.056601. 
[24] M. L. Cooper et al., "Waveguide dispersion effects in silicon-oninsulator coupled-resonator optical waveguides," Opt. Lett., vol. 35, no. 18, p. 3030, Sep. 2010, doi: 10.1364/OL.35.003030.

[25] S. Mookherjea, D. S. Cohen, and A. Yariv, "Nonlinear dispersion in a coupled-resonator optical waveguide," Opt. Lett., vol. 27, no. 11, p. 933, Jun. 2002, doi: 10.1364/OL.27.000933.

[26] J. E. Heebner, R. W. Boyd, and Q. H. Park, "Slow light, induced dispersion, enhanced nonlinearity, and optical solitons in a resonator-array waveguide," Phys. Rev. E, vol. 65, no. 3, p. 036619, 2002.

[27] R. Aguinaldo et al., "Wideband silicon-photonic thermo-optic switch in a wavelength-division multiplexed ring network," Opt. Express, vol. 22, no. 7, p. 8205, Apr. 2014, doi: 10.1364/OE.22.008205.

[28] X. Wang, P. O. Weigel, J. Zhao, M. Ruesing, and S. Mookherjea, "Achieving beyond-100-GHz large-signal modulation bandwidth in hybrid silicon photonics Mach Zehnder modulators using thin film lithium niobate," APL Photonics, vol. 4, no. 9, p. 096101, Sep. 2019, doi: $10.1063 / 1.5115243$.

[29] S. Mookherjea, J. R. Ong, X. Luo, and L. Guo-Qiang, "Electronic control of optical Anderson localization modes," Nature Nanotech, vol. 9, no. 5, pp. 365-371, May 2014, doi: 10.1038/nnano.2014.53.

[30] J. R. Ong, R. Kumar, and S. Mookherjea, "Silicon microring-based wavelength converter with integrated pump and signal suppression," Opt. Lett., vol. 39, no. 15, p. 4439, Aug. 2014, doi: 10.1364/OL.39.004439.

[31] X. Wang et al., "Wide-range and fast thermally-tunable silicon photonic microring resonators using the junction field effect," Opt. Express, vol. 24, no. 20, p. 23081, Oct. 2016, doi: 10.1364/OE.24.023081.

[32] J. Zhao, C. Ma, M. Rüsing, and S. Mookherjea, "High Quality Entangled Photon Pair Generation in Periodically Poled Thin-Film Lithium Niobate Waveguides," Phys. Rev. Lett., vol. 124, no. 16, p. 163603, Apr. 2020, doi: 10.1103/PhysRevLett.124.163603.

[33] J. R. Ong and S. Mookherjea, "Quantum light generation on a silicon chip using waveguides and resonators," Opt. Express, vol. 21, no. 4, p. 5171, Feb. 2013, doi: 10.1364/OE.21.005171.

[34] M. Savanier, R. Kumar, and S. Mookherjea, "Optimizing photon-pair generation electronically using a $p-i-n$ diode incorporated in a silicon microring resonator," Appl. Phys. Lett., vol. 107, no. 13, p. 131101, Sep. 2015, doi: 10.1063/1.4932047.

[35] C. Ma, X. Wang, V. Anant, A. D. Beyer, M. D. Shaw, and S. Mookherjea, "Silicon photonic entangled photon-pair and heralded single photon generation with CAR $>12,000$ and $\mathrm{g}^{\wedge}(2)(0)<0006$," Opt. Express, vol. 25, no. 26, p. 32995, Dec. 2017, doi: 10.1364/OE.25.032995. 
[36] R. Kumar, M. Savanier, J. R. Ong, and S. Mookherjea, "Entanglement measurement of a coupled silicon microring photon pair source," Opt.

Express, vol. 23, no. 15, p. 19318, Jul. 2015, doi: 10.1364/OE.23.019318.

[37] A. Ciattoni, B. Crosignani, S. Mookherjea, and A. Yariv, "Nonparaxial dark solitons in optical Kerr media," Opt. Lett., vol. 30, no. 5, p. 516, Mar. 2005, doi: 10.1364/OL.30.000516. 